\title{
Yamaguchi Facial Expression-Making Task in Alzheimer's Disease: A Novel and Enjoyable Make-a-Face Game
}

\author{
Tomoharu Yamaguchi ${ }^{\mathrm{a}, \mathrm{b}}$ Yohko Maki ${ }^{\mathrm{a}, \mathrm{c}}$ Haruyasu Yamaguchi ${ }^{\mathrm{a}}$ \\ ${ }^{a}$ Gunma University Graduate School of Health Sciences, ${ }^{b}$ Department of Rehabilitation, Gunma \\ University of Health and Welfare, and ' ${ }^{\complement}$ Geriatrics Research Institute and Hospital, Maebashi, Japan
}

\section{Key Words}

Dementia - Facial expression - Dementia care - Caregivers - Emotional reactions to dementia • Cognitive tests $\cdot$ Behavioral/psychiatric symptoms of dementia

\begin{abstract}
Background: To assess the ability to make emotional facial expressions, we newly developed the Yamaguchi facial expression-making task (Y-FEMT). Method: We recruited 20 normal controls and 61 outpatients: 10 with amnestic mild cognitive impairment (aMCl), 34 with mild Alzheimer's disease (AD), and 17 with moderate AD. In the Y-FEMT, smile and anger expressions were made by arranging face parts. We examined the relationship between each Y-FEMT score and the Mini-Mental State Examination (MMSE) score or overlapping figure identification test (Fig-test). Results: The Total score (0-20) was nearly achieved in controls (18.9 \pm 1.4$)$ and declined with AD progression ( $\mathrm{aMCl} 17.2 \pm 2.4$, mild $\mathrm{AD} 15.7 \pm 2.6$, moderate $\mathrm{AD} 12.3 \pm 2.7)$. The Anger score (0-10) was significantly lower than the Smile score (0-10) in mild and moderate AD ( $p=0.007$ and $p=0.006$, respectively). The Structure score (0-6 each) correlated well with both the MMSE score $(r=0.44, p<0.001)$ and Fig-test $(r=0.45, p<0.001)$, whereas the Expression score ( $0-4$ each) correlated only with the MMSE score $(r=0.33, p=0.01)$. The Subjective scores $(0-4)$, evaluated by 10 therapists, highly correlated with the Total score. Additionally, the Y-FEMT promoted laughter and a convivial atmosphere. Conclusion: The Y-FEMT pleasantly assessed the ability to make emotional facial expressions without special equipment. Furthermore, the Y-FEMT may provide helpful clues for caregivers to achieve good communication with AD patients for better care.




\section{Introduction}

In the care of Alzheimer's disease (AD) patients, deficits in the recognition of emotional facial expressions may cause problems between $\mathrm{AD}$ patients and their caregivers because emotion perception deficits, rather than general cognition, have been linked to interpersonal difficulties $[1,2]$. Furthermore, problems in emotional perception, but not cognitive function or mood, predict quality of life [3]. Patients with AD have deficits in recognizing facial emotion, which may be independent of their impairment in recognizing non-emotional features of faces [4]. Discriminating facial identities has been shown to be impaired in AD patients [5]. Recognition of emotional facial expressions can be impaired in mild cognitive impairment $(\mathrm{MCI})$ prior to the diagnosis of $\mathrm{AD}[4,6]$. In addition, the ability to recognize emotional facial expressions is impaired with the progression of $\mathrm{AD}$ [7], but recognition and reaction to emotional facial stimuli are partially preserved in the advanced stages of dementia, in which the most sensitively identified emotion is happiness [8].

As described above, there are many reports on disturbed recognition of emotional facial expressions in $\mathrm{MCI}$ and $\mathrm{AD}$. However, no study has investigated the ability of AD patients to make facial expressions. Furthermore, it is not easy to detect the disturbed recognition of emotional facial expression in a clinical setting because special techniques or instruments, such as a computer with morphing technology, are needed for detection.

We thus developed a new cognitive task, the Yamaguchi facial expression-making task (Y-FEMT), to assess the ability of patients to make emotional facial expressions by arranging pieces of face parts. The origin of the Y-FEMT is the traditional Japanese make-a-face game 'Fuku-warai', meaning 'Lucky Laugh'. The aim of this game is to put face parts (e.g. eyebrows, eyes, nose and mouth) on a face outline while the player's eyes are covered with a hand towel. Then, all participants enjoy laughing at the funniness of these facial expressions. Fukuwarai is traditionally played during the New Year's holidays. The Y-FEMT consisted of two tasks to make up 'Smile' and 'Anger' faces without a blindfold. We describe the development of the Y-FEMT, how we used to investigate patients' ability to make emotional facial expression, and its association with other cognitive tests. After defining the objective standard of the Y-FEMT, we added a subjective scoring method of the Y-FEMT for easy use in clinical practice.

\section{Methods}

\section{Participants}

Normal controls (NC; $\mathrm{n}=20$ ), aged 61 to 80 years, were recruited from community dwellers who participated in the 'Prevention of mental decline' project in Takasaki City, Gunma, Japan. These participants were judged normal based on the results of cognitive tests and medical interviews by clinicians specializing in dementia. We recruited 61 participants who were diagnosed as having amnestic MCI (aMCI) or AD at the outpatient clinic of the Geriatrics Research Institute and Hospital in Maebashi, Gunma, Japan.

The AD patients were diagnosed based on the criteria of the National Institute of Neurological and Communicative Disorders and Stroke and Alzheimer's Disorders and Related Disorders Association (NINCDS-ADRDA) [9]. Similarly, the aMCI patients were diagnosed based on a previous study [10]. In the present study, 61 participants were classified according to the Clinical Dementia Rating (CDR) by an experienced neurologist. The discriminant criteria of mild and moderate AD were CDR $1(n=34)$ and CDR $2(n=17)$, respectively.

None of the participants demonstrated other psychiatric disorders or had problems with alcoholism, motor deficits such as paralysis, major heart disease or neurological or psychi- 
Table 1. Demographics and clinical characteristics

\begin{tabular}{|c|c|c|c|c|c|}
\hline & $\begin{array}{l}\text { All } \\
(\mathrm{n}=81)\end{array}$ & $\begin{array}{l}\mathrm{NC} \\
(\mathrm{n}=20)\end{array}$ & $\begin{array}{l}\mathrm{aMCI} \\
(\mathrm{n}=10)\end{array}$ & $\begin{array}{l}\text { Mild AD } \\
(\mathrm{n}=34)\end{array}$ & $\begin{array}{l}\text { Moderate AD } \\
(\mathrm{n}=17)\end{array}$ \\
\hline Male/female & $27 / 54$ & $7 / 13$ & $6 / 4$ & $12 / 22$ & $2 / 15$ \\
\hline Age, years & $77.5 \pm 6.8$ & $72.3 \pm 4.9$ & $74.3 \pm 7.0$ & $79.6 \pm 6.6$ & $81.3 \pm 4.4$ \\
\hline Education, years & $10.3 \pm 2.7$ & $12.5 \pm 2.0$ & $10.6 \pm 4.0$ & $9.5 \pm 2.1$ & $9.2 \pm 2.4$ \\
\hline MMSE score & $21.6 \pm 6.5$ & $28.8 \pm 1.4$ & $26.5 \pm 2.6$ & $20.2 \pm 3.2$ & $12.9 \pm 4.2$ \\
\hline \multicolumn{6}{|c|}{ Y-FEMT score (objective) } \\
\hline Total & $16.0 \pm 3.2$ & $18.9 \pm 1.4$ & $17.2 \pm 2.4$ & $15.7 \pm 2.6$ & $12.3 \pm 2.7$ \\
\hline Smile & $8.4 \pm 1.6$ & $9.6 \pm 0.9$ & $9.0 \pm 1.2$ & $8.4 \pm 1.6$ & $6.9 \pm 1.2$ \\
\hline Anger & $7.5 \pm 2.1 * * *$ & $9.3 \pm 1.1$ n.s. & $8.2 \pm 1.6$ n.s. & $7.4 \pm 1.7^{* *}$ & $5.4 \pm 2.0 * *$ \\
\hline Structure & $10.4 \pm 1.8$ & $11.6 \pm 0.8$ & $11.0 \pm 1.1$ & $10.6 \pm 1.3$ & $8.5 \pm 2.3$ \\
\hline Expression & $5.5 \pm 2.0$ & $7.3 \pm 0.9$ & $6.2 \pm 1.9$ & $5.1 \pm 1.8$ & $3.8 \pm 1.3$ \\
\hline Subjective score ${ }^{\#}$ & $2.8 \pm 1.1$ & $3.5 \pm 0.6$ & $3.3 \pm 0.8$ & $2.8 \pm 1.0$ & $1.8 \pm 1.1$ \\
\hline
\end{tabular}

Data are presented as mean \pm standard deviation. n.s. $=$ Not significant.

${ }^{* *} \mathrm{p}<0.01,{ }^{* * *} \mathrm{p}<0.001$ : comparison between the Smile and Anger scores by a paired $\mathrm{t}$ test. ${ }^{*}$ Subjective score was assessed by 10 evaluators.

atric disorders other than the primary diagnosis of AD or aMCI. Demographic data and clinical characteristics are shown in table 1. All participants reported normal or correctedto-normal vision, and they were naive with regard to the purpose of the experiment. The Ethics Board of Gunma University School of Health Sciences approved all procedures (No. 21-47), and signed informed consent was obtained.

All participants underwent the Mini-Mental State Examination (MMSE) [11] and overlapping figure identification test (Fig-test), which consists of line-drawn figures of six objects: hat, eyeglasses, vase, apple, tulip and hammer. The participants were asked to identify each figure, and the score range was $0-6$.

\section{Procedure}

The Y-FEMT contains two tasks. Participants were asked to arrange parts of a face puzzle (fig. 1a) to make two kinds of emotional facial expressions, 'Smile' and 'Anger' (fig. 1b, c). The face puzzle used in this study was made of thick, stiff paper and consisted of one outline plate of a face with hair $(25 \mathrm{~cm}$ long and $19 \mathrm{~cm}$ width) and six facial parts: two eyebrows (or mustache; $5.5 \mathrm{~cm}$ long and $2 \mathrm{~cm}$ width), two eyes $(6.5 \mathrm{~cm}$ long and $3.5 \mathrm{~cm}$ width), one nose (4.5 cm long and $4 \mathrm{~cm}$ width) and one mouth (10 cm long and $3.5 \mathrm{~cm}$ width) (fig. 1a).

\section{Protocol}

The protocol was as follows: (1) The participant sits at their desk. The face outline plate (fig. 1a) is placed in the center of the desk. (2) The Y-FEMT starts with the Smile task (fig. 1b) followed by the Anger task (fig. 1c). (3) For the Smile task, the examiner hands six randomly-oriented parts of a human face (fig. 1a) to the participant, and gives the simple instruction: 'You must use all of these parts and arrange the pieces to make a smiling face'. The instruction can be repeated, if required. (4) Then, the participant arranges the face parts on the face outline plate to make a smiling face. (5) The examiner records the completed task by taking a photograph (fig. 1d-f). If the participant cannot complete the task within $2 \mathrm{~min}$, the uncompleted task is scored. (6) Avoid giving advice or pointing out any error until the end of the next task. (7) For the 'Anger' task, the examiner hands the participants six face 


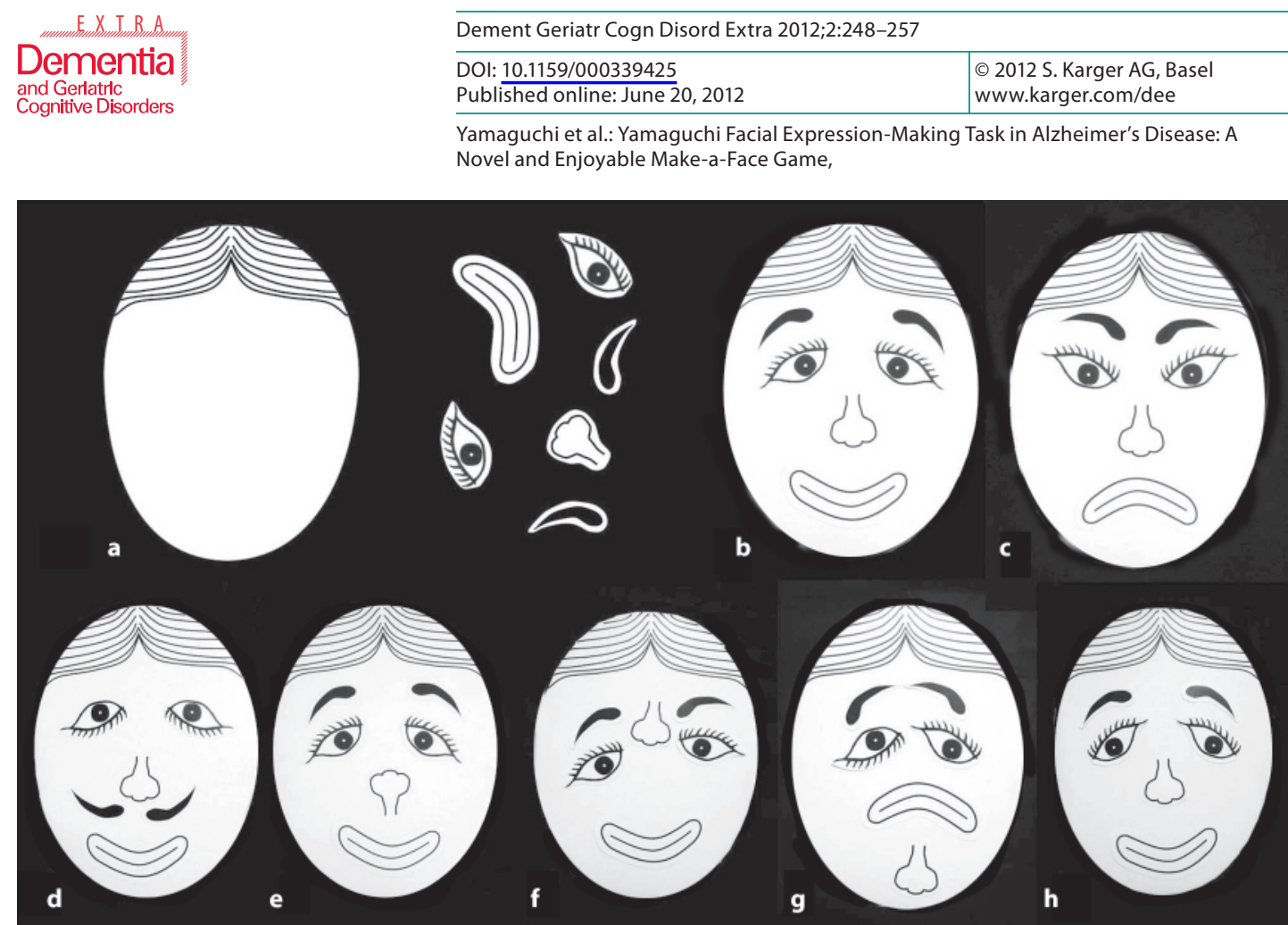

Fig. 1. Parts of the Y-FEMT, exemplary results and error patterns. a The Y-FEMT consists of one outline plate and six simple parts of the human face. Exemplary results of the Smile task (b; Smile score was 10/10) and Anger task (c; Anger score was 10/10) in healthy elderly people. d-f Examples of the Smile task in AD patients. The score was decreased by 1 point for incorrect orientation of the eyes, but adopting the eyebrows as a moustache was not considered a mistake (d; Smile score was 9/10). Orientation of the nose is upside-down (e; Smile score was 9/10). Placement of the nose and symmetric property are different and expression score was 3 (f; Smile score was 7/10). g, h Examples of the Anger task in mild-to-moderate AD patients. Only the position of the eyebrows and eyes are correct, each gaining 1 point (g; Anger score was $2 / 10$ ), and the fundamental structure of the face was good, except that the orientation of the eyes is leftright reversed, but the facial expression is different (h; Anger score was 5/10).

parts and gives a similar instruction: 'You must use all of these parts and arrange the pieces to look like an angry face'. (8) The participant arranges the six parts on the face outline to make an angry face. (9) The examiner records the completed task by taking a photograph (fig. 1g, h).

There is the option to look at the photos and laugh together after finishing both tasks.

\section{Guideline for Objective Scoring (table 2)}

Structure Score

Assessment of the fundamental structure of the face (0-12 points; $0-6$ points each for Smile and Anger structures):

- Eyebrows are judged for placement in the uppermost part of the face and above the eyes or not (1 point). If participant perceived the eyebrows as a mustache, judge whether they are placed between the mouth and nose, and placed on the lower face ( 1 point). Point allocation is $0-1$.

- Eyes are judged for placement and orientation. The criteria for placement of eyes is whether the pupil of the left eye is in the upper left quarter of the face and the pupil of the right eye is in the upper right quarter of the face (1 point). Orientation of the eyes is assessed for left-right reversal or upside-down (1 point). Therefore, point allocation for the eyes is $0-2$. 
Table 2. Instructions for scoring

\begin{tabular}{|c|c|c|c|}
\hline & Sum & $\begin{array}{l}\text { Smile } \\
\text { task }\end{array}$ & $\begin{array}{l}\text { Anger } \\
\text { task }\end{array}$ \\
\hline \multicolumn{4}{|l|}{ Objective scoring } \\
\hline Structure score for each Smile and Anger task & $0-12$ & & \\
\hline Eyebrows or mustache (uppermost part of face or lie between nose and mouth) & & $0-1$ & $0-1$ \\
\hline Placement of eyes (pupil is in the upper left or right quarter of face) & & $0-1$ & $0-1$ \\
\hline Orientation of eyes (no left-right reversal or upside-down placement) & & $0-1$ & $0-1$ \\
\hline Placement and orientation of nose (around the center and under eyes) & & $0-1$ & $0-1$ \\
\hline Placement and orientation of mouth (bottommost part of face) & & $0-1$ & $0-1$ \\
\hline Symmetric property (bilaterally symmetric placement) & & $0-1$ & $0-1$ \\
\hline Expression score for Smile task/Anger task & $0-8$ & & \\
\hline Outer corners of eyes slant downward/upward (each eye $0-1$ ) & & $0-2$ & $0-2$ \\
\hline Outer corners of mouth slant upward/downward & & 0 or 2 & 0 or 2 \\
\hline Total score (sum of Smile score and Anger score) & $0-20$ & & \\
\hline Smile score and Anger score are the sum of each structure score and expression score & & $0-10$ & $0-10$ \\
\hline \multicolumn{4}{|l|}{ Subjective scoring } \\
\hline Subjective score for each Smile and Anger task & $0-4$ & & \\
\hline Subjective evaluations: quite good $=2$, approximate $=1$, different $=0$ & & $0-2$ & $0-2$ \\
\hline
\end{tabular}

- Appropriate placement of the nose is within one-half of the distance from the center of the face to the outline of the face and located between eyes and mouth. When both placement and orientation are appropriate, the score for the nose is 1 point.

- Appropriate placement of the mouth is as the bottommost of the face parts. Appropriate orientation of the mouth is with the length in the horizontal direction and the slant of the mouth within 45 degrees, regardless of whether it is upside-down. When both placement and orientation are appropriate, the score for the mouth is 1 point.

- Symmetric property (1 point) is given for bilaterally symmetric placement of the face parts.

- The 'Structure' score (0-12 points) is the sum of both the Smile and Anger structures (0-6 points each).

\section{Expression Score}

Assessment of facial expression (0-8 points; 0 -4 points each for Smile and Anger expressions):

- For the Smile task, the outer corners of each eye must slant downward (each eye 1 point) and the outer corners of the mouth must slant upward (2 points).

- For the Anger task, the outer corners of each eye must slant upward (each eye 1 point) and the outer corners of the mouth must slant downward ( 2 points). These directions are the opposite of those in the Smile task. Eyebrows (or mustache) and nose are exempt from directional judgment. However, if there is an error in the placement of the eyes or mouth, it is not pointed out.

\section{Smile Score and Anger Score}

The Smile and Anger scores are the summations of the Structure score and the Expression score for each task. Each point allocation is $0-10$. 
Total Score

The Total score is the sum of the Smile and Anger scores, which are equal to the sums of the Structure scores and the Expression scores. Point allocation is 0-20.

Guideline for Subjective Scoring (Point Allocation 0-2 for Each Task)

If an evaluator thinks each facial expression is quite appropriate, the score is 2 , and if it is approximately appropriate or totally different, the score is 1 or 0 , respectively. In this study, the facial expressions in each task were assessed by 10 evaluators, who were experienced occupational therapists.

Data Analyses

We scored the pictures created by all participants. To investigate group differences of objective Y-FEMT scores, we carried out analyses of covariance (ANCOVA) with covariates of age and educational years. Post hoc analysis for each group was conducted with Bonferroni correction in all 81 participants. We examined the relationship between each objective Y-FEMT score and MMSE or Fig-test scores by Pearson's product-moment correlation coefficient in the 61 patients. The influence of the order of tasks (c.f. protocol) was analyzed in the $20 \mathrm{NC}$ participants. Test-retest reliability (test-retest interval $127.4 \pm 51.4$ days) was examined in 22 mild-to-moderate AD patients (age $80.0 \pm 7.4$ years, education $9.5 \pm 1.9$ years, MMSE score $17.1 \pm 5.4 ; 13$ mild AD and 9 moderate AD patients). Furthermore, we examined the relationship between the objective Y-FEMT score and the Subjective score assessed by 10 experienced therapists, and investigated inter-rater reliability by intraclass correlation coefficients (ICC) and correlation with the objective Y-FEMT score for clinical utility. All statistical analyses were performed with the Japanese version of SPSS 19.0 for Windows (IBM Com.). Results are reported at a significance level of $\mathrm{p}<0.05$.

\section{Results}

\section{Y-FEMT in Normal Controls}

Demographic data and the results of the Y-FEMT in NC $(n=20)$ are shown in table 1. In NC, the total Y-FEMT score was nearly achieved, although some participants showed a decreased Anger score. To analyze the influence of the order of the tasks, NC were randomly divided into two groups: 'Smile-Anger' group $(\mathrm{n}=8$, age $72.8 \pm 5.7$ years, education 12.1 \pm 2.2 years, MMSE score $28.5 \pm 1.2)$ and 'Anger-Smile' group $(\mathrm{n}=12$, age $72.0 \pm 4.5$ years, education $12.8 \pm 1.9$ years, MMSE score $29.0 \pm 1.5$ ). The task order did not influence the scores $(\mathrm{p}=0.578$, Student's t test; online suppl. table 1 ; for all online suppl. material, see www. karger.com/doi/10.1159/000339425).

\section{Making Emotional Facial Expressions and Relationship with Disease Progression}

All 81 participants accomplished the two tasks within the 2-min time limit. Results and representative faces made during the tasks are presented in table 1 and figure 1, respectively. In all participants, the Total score decreased with the progression of $\mathrm{AD}$, as demonstrated by ANCOVA with covariates of age and educational years $(F(5,75)=17.08$, p < 0.001). The results of the post hoc analysis with Bonferroni correction indicated a significant difference only between mild and moderate AD $(\mathrm{p}<0.001)$. Some strange and funny faces were created by AD patients, as shown in figure 1d-h. Smile, Anger and Structure scores were significantly different between mild and moderate AD patients $(p<0.01, p<$ 0.01 and $p<0.001$, respectively), but not between NC and aMCI patients, or between aMCI and mild AD patients. The Expression score was significantly different between NC and 

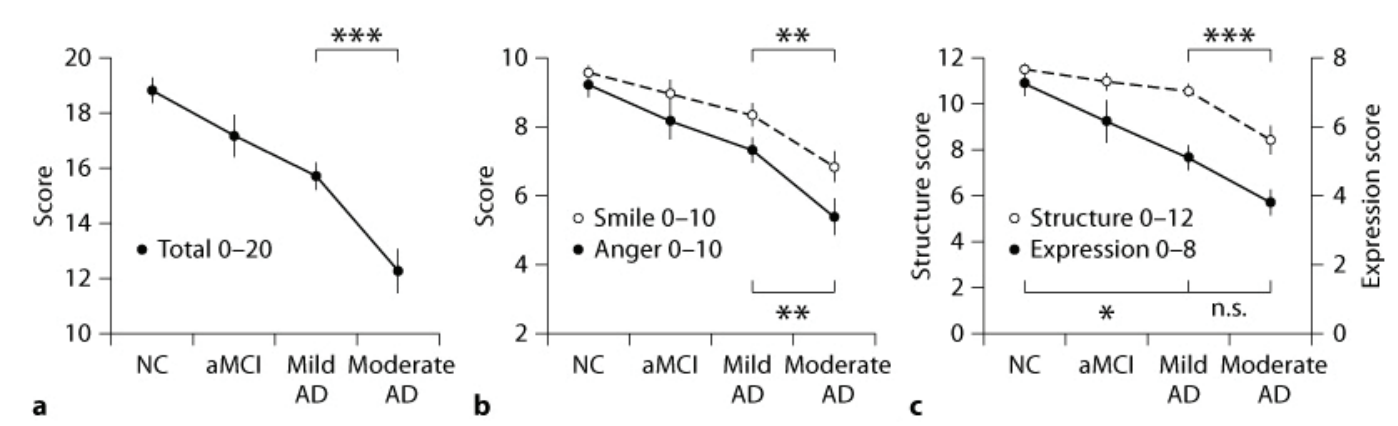

Fig. 2. Y-FEMT scores in four groups. Numbers on the graph indicate mean \pm SE. Point allocation for the Total score is $0-20(\mathbf{a})$, for the Smile score and Anger score it is $0-10(\mathbf{b})$, for the Structure score 0-12 and for the Expression score it is $0-8$ (c). All scores decreased with the progression of AD. There was a significant difference between mild and moderate AD except for the Expression score. ${ }^{*} \mathrm{p}<0.05,{ }^{* *} \mathrm{p}<$ $0.01,{ }^{* * *} \mathrm{p}<0.001 ; \mathrm{n} . \mathrm{s} .=$ Not significant. ANCOVA with covariates of age and educational years.

aMCI patients $(\mathrm{p}=0.01)$, but not between mild AD and moderate AD patients $(\mathrm{p}=0.069)$. Thus, objective Y-FEMT scores decreased with the progression of AD especially in moderate $\mathrm{AD}$ patients.

\section{Smile Score versus Anger score, and Structure Score versus Expression Score}

The comparison between the Smile score and Anger score for all participants demonstrated that the Smile score was significantly higher than the Anger score $(\mathrm{p}<0.001$, paired $\mathrm{t}$ test; table 1). In the analysis of each group, the Smile score and Anger score were not significantly different in the NC or aMCI groups $(\mathrm{p}=0.286, \mathrm{n}=20$ and $\mathrm{p}=0.137, \mathrm{n}=10$, respectively), while the Smile score was significantly higher than the Anger score in both the mild $\mathrm{AD}$ and moderate $\mathrm{AD}$ groups by paired test $(\mathrm{p}=0.007, \mathrm{n}=34$ and $\mathrm{p}=0.006, \mathrm{n}=17$, respectively; table 1). Thus, the $\mathrm{AD}$ participants achieved a higher score for the Smile task than for the Anger task (fig. 2b).

The Structure score and Expression score also declined with the progression of AD (table 1; fig. 2c). In the four groups, both Expression and Structure scores showed significant differences $(F(5,75)=11.08, \mathrm{p}<0.001$ and $\mathrm{F}(5,75)=10.27, \mathrm{p}<0.001$, respectively). Post hoc analysis with Bonferroni correction indicated that the Expression score significantly decreased between NC and mild AD ( $\mathrm{p}=0.01)$, and that the Structure score significantly decreased between mild AD and moderate AD. Thus, the Expression score declined earlier than the Structure score.

\section{Relationship between Expression-Making Tasks and Cognitive Tests}

Next, we examined the relationship between Y-FEMT scores and two cognitive tests, the MMSE and Fig-test, in participants other than NC $(n=61)$ by Pearson's product-moment correlation coefficient. On analysis of the correlation between Y-FEMT and MMSE scores, each Y-FEMT score was mild-to-moderately and significantly correlated with the MMSE score (Total $\mathrm{r}=0.463, \mathrm{p}<0.001$; Smile $\mathrm{r}=0.346, \mathrm{p}=0.006$; Anger $\mathrm{r}=0.434, \mathrm{p}<$ 0.001 ; Structure $\mathrm{r}=0.440, \mathrm{p}<0.001$, and Expression $\mathrm{r}=0.328, \mathrm{p}=0.01$; online suppl. table 2). On analysis of the correlation between Y-FEMT and Fig-test scores, Y-FEMT scores other than the Expression score were mild-to-moderately correlated with the Fig-test score (Total $\mathrm{r}=0.387, \mathrm{p}=0.002$; Smile $\mathrm{r}=0.357, \mathrm{p}=0.005$; Anger $\mathrm{r}=0.308, \mathrm{p}=0.016$, and Structure $\mathrm{r}=$ 
$0.452, \mathrm{p}<0.001)$. Note that the Expression score was not significantly correlated with the Fig-test score $(\mathrm{r}=0.189, \mathrm{p}=0.145)$, but was significantly correlated with the MMSE score. In contrast, the Structure score showed significant correlations with both the MMSE and Fig-test scores.

\section{Test-Retest Reliability of the Y-FEMT}

The test-retest reliability was based on the ratings of 22 mild-to-moderate $\mathrm{AD}$ patients. The intra-rater reliability of the Total score was sufficiently high $(\operatorname{ICC}(1.1)=0.927, \mathrm{p}<0.001)$. Similarly to the analysis of correlation between the first and second tries, the Total score showed a high correlation ( $\mathrm{r}=0.936, \mathrm{p}<0.001$, Pearson's correlation).

\section{Subjective Score of the Y-FEMT for Clinical Practice}

The inter-rater reliability of the Subjective scores $(n=81)$ by 10 evaluators was good $(\operatorname{ICC}(2,1)=0.760, \mathrm{p}<0.001)$. Furthermore, the Subjective scores showed a high correlation with both the Total and Expression scores $(\mathrm{r}=0.789, \mathrm{p}<0.001$ and $\mathrm{r}=0.770, \mathrm{p}<0.001$, respectively).

\section{Discussion}

\section{Comparison with Previous Studies}

This study investigated how the ability to make emotional facial expressions changes with the progression of AD. We developed the Y-FEMT for this purpose.

In the objective scoring, the Total score of the Y-FEMT decreased with the progression of $\mathrm{AD}$. This finding about the ability to make emotional facial expressions is in agreement with results from previous studies on the recognition of emotional facial expressions, which start to become impaired in MCI $[6,12]$ and then become more severely impaired with the progression of $\mathrm{AD}[7,13,14]$.

Comparing the Structure score and Expression score, the Structure score decreased between mild and moderate $\mathrm{AD}$, whereas the Expression score started to decrease between NC and mild $\mathrm{AD}$. This finding suggests that the ability to make the fundamental structure of the face is maintained in the early stages of AD. Furthermore, the Structure score was significantly and moderately correlated with the Fig-test score, whereas the Expression score did not correlate with the Fig-test score. This suggests that the ability to make the fundamental structure of the face is closely related to visual function, which is impaired in the later stages of $\mathrm{AD}$.

Comparing the Smile score and Anger score, the Anger score was significantly lower than the Smile score in AD patients. This findings agree with those from previous studies on the recognition of facial expressions, in which a happy face was most easily recognized cross-culturally (both Japanese and Caucasian) [15] among both healthy people and elderly subjects with $\mathrm{AD}[7,12,16,17]$, as well as people with severe dementia [8]. In addition, the coefficient of correlation between the MMSE and Anger scores $(r=0.434)$ tended to be higher than that between the MMSE and Smile scores $(r=0.346)$. Thus, the findings of this study suggest that the ability to make an angry face is impaired in parallel with general cognitive function, whereas the ability to make a smiling face is maintained for longer.

\section{Utility of the Y-FEMT}

Most previous studies on emotional facial expression examined the ability to recognize computer-created faces. However, the Y-FEMT assesses the ability to make the fundamental structures of the face as well as emotional facial expressions. Most participants responded 
Novel and Enjoyable Make-a-Face Game,

when completing the Y-FEMT. They said 'I'm not great with games like this' but smiled happily while performing the tasks. In addition, the Y-FEMT induced laughter and a convivial atmosphere, especially when we praised the participants' results after finishing the two tasks. This is a primary characteristic of the traditional Japanese game 'Fuku-warai'. In 'Fuku-warai', the resultant facial expressions are comical and funny, because the game is played while blindfolded. When dementia patients performed the Y-FEMT without a blindfold, they also created comical faces and everyone including themselves could laugh (fig. 1d-h). However, their family caregivers showed various responses; some were shocked and disappointed on seeing the results or reprehended the patients' mistakes, while other caregivers had fun together with a warm smile even if the patients scored poorly on the tasks. We can estimate some common treatment or communication problems between caregivers and patients based on the Y-FEMT, whether caregivers accept or become angry with the patients' mistakes. Thus, the Y-FEMT is a useful tool for family education on appropriate care because emotional communication and support are very important in dementia care [8]. Problems with emotion perception or emotion comprehension, rather than deterioration of cognitive function and mood, influence the quality of life and problems of interpersonal behaviors in AD patients $[1,3]$. Thus, it is better for caregivers to understand the level of social cognition of $\mathrm{AD}$ patients. The Y-FEMT provides helpful clues for caregivers to understand the ability of the $\mathrm{AD}$ patients to make emotional facial expressions.

In addition, the Y-FEMT is a convenient task for assessing the ability to make faces showing expression and fundamental structure, and is less stressful than conventional cognitive tests. It is easy to do, simple to understand the rules, takes a short time, has non-committal answers and protects patients from a feeling of 'failure'. Furthermore, the Y-FEMT does not require special instruments such as a computer or monitor, unlike other recently published methods.

In this study, the Subjective score correlated highly with the objective Y-FEMT scores, Total score and Expression score. Moreover, the inter-rater reliability of the Subjective score was sufficiently high. Thus, objective scoring can be replaced by the Subjective score in clinical practice. We hope that the Y-FEMT will provide helpful clues for caregivers to improve care and communication with AD patients, and that the task performance will be enjoyable for both the patients and their caregivers.

\section{Acknowledgements}

We thank the participants in this study, as well as their relatives, for their contribution.

This work was conducted without funding. However, H. Yamaguchi is supported by a Grant-in-Aid for Scientific Research from the Ministry of Education, Science, Sports, Culture and Technology, Japan (23300197 and 22650123), and a Grant-in-Aid for Scientific Research (H22-Ninchisho-Ippan-004) from the Ministry of Health, Labor and Welfare of Japan. T. Yamaguchi is supported by a grant-in-aid from the Asawo Health Promotion Foundation, Gunma.

\section{Disclosure Statement}

The authors have no conflict of interest to declare. 


\section{References}

-1 Shimokawa A, Yatomi N, Anamizu S, Torii S, Isono H, Sugai Y, Kohno M: Influence of deteriorating ability of emotional comprehension on interpersonal behavior in Alzheimer-type dementia. Brain Cogn 2001;47:423-433.

2 Gruetzner H: Alzheimer's: A Caregiver's Guide and Sourcebook, ed 3. New York, John Wiley and Sons, Inc, 2001.

3 Phillips L, Scott C, Henry J, Mowat D, Bell J: Emotion perception in Alzheimer's disease and mood disorder in old age. Psychol Aging 2010;25:38-47.

-4 Hargrave R, Maddock RJ, Stone V: Impaired recognition of facial expressions of emotion in Alzheimer's disease. J Neuropsychiatry Clin Neurosci 2002;14:64-71.

-5 Roudier M, Marcie P, Grancher AS, Tzortzis C, Starkstein S, Boller F: Discrimination of facial identity and of emotions in Alzheimer's disease. J Neurol Sci 1998;154:151-158.

-6 Teng E, Lu PH, Cummings JL: Deficits in facial emotion processing in mild cognitive impairment. Dement Geriatr Cogn Disord 2007;23:271-279.

-7 Weiss EM, Kohler CG, Vonbank J, Stadelmann E, Kemmler G, Hinterhuber H, Marksteiner J: Impairment in emotion recognition abilities in patients with mild cognitive impairment, early and moderate Alzheimer disease compared with healthy comparison subjects. Am J Geriatr Psychiatry 2008; 16:974-980.

-8 Guaita A, Malnati M, Vaccaro R, Pezzati R, Marcionetti J, Vitali S, Colombo M: Impaired facial emotion recognition and preserved reactivity to facial expressions in people with severe dementia. Arch Gerontol Geriatr 2009;49(suppl 1):135-146.

-9 Dubois B, Feldman HH, Jacova C, Dekosky ST, Barberger-Gateau P, Cummings J, Delacourte A, Galasko D, Gauthier S, Jicha G, Meguro K, O’Brien J, Pasquier F, Robert P, Rossor M, Salloway S, Stern Y, Visser PJ, Scheltens P: Research criteria for the diagnosis of Alzheimer's disease: revising the NINCDS-ADRDA criteria. Lancet Neurol 2007;6:734-746.

-10 Petersen RC, Parisi JE, Dickson DW, Johnson KA, Knopman DS, Boeve BF, Jicha GA, Ivnik RJ, Smith GE, Tangalos EG, Braak H, Kokmen E: Neuropathologic features of amnestic mild cognitive impairment. Arch Neurol 2006;63:665-672.

-11 Folstein M, Folstein S, McHugh P: 'Mini-mental state'. A practical method for grading the cognitive state of patients for the clinician. J Psychiatr Res 1975;12:189-198.

12 Spoletini I, Marra C, Di Iulio F, Gianni W, Sancesario G, Giubilei F, Trequattrini A, Bria P, Caltagirone C, Spalletta G: Facial emotion recognition deficit in amnestic mild cognitive impairment and Alzheimer disease. Am J Geriatr Psychiatry 2008;16:389-398.

-13 Bediou B: Impaired social cognition in mild Alzheimer disease. J Geriatr Psychiatry Neurol 2009;22: 130-140.

-14 Lavenu I, Pasquier F: Perception of emotion on faces in frontotemporal dementia and Alzheimer's disease: a longitudinal study. Dement Geriatr Cogn Disord 2005;19:37-41.

-15 Shioiri T, Someya T, Helmeste D, Tang SW: Misinterpretation of facial expression: a cross-cultural study. Psychiatry Clin Neurosci 1999;53:45-50.

-16 Burnham H, Hogervorst E: Recognition of facial expressions of emotion by patients with dementia of the Alzheimer type. Dement Geriatr Cogn Disord 2004;18:75-79.

-17 García-Rodríguez B, Fusari A, Rodríguez B, Hernández J, Ellgring H: Differential patterns of implicit emotional processing in Alzheimer's disease and healthy aging. J Alzheimers Dis 2009;18: $541-551$. 